Ciência Florestal, Santa Maria, v. 26, n. 4, p. 285-293, jan.-mar., 2016

ISSN 0103-9954

\title{
SUBSTRATOS E TEMPERATURAS PARA TESTE DE GERMINAÇÃO EM SEMENTES DE MYRTACEAE
}

\author{
SUBSTRATES AND TEMPERATURES FOR GERMINATION TEST OF MYRTACEAE SEEDS
}

Juliano Pereira Gomes ${ }^{1}$ Luciana Magda de Oliveira ${ }^{2}$ Paula Iaschitzki Ferreira ${ }^{3}$ Felipe Batista $^{4}$

\section{RESUMO}

As espécies brasileiras de Myrtaceae compreendem diversas plantas arbóreas e arbustivas que podem ser utilizadas na produção de frutos para consumo in natura ou para industrialização. Informações sobre avaliação da qualidade de sementes dessas espécies não estão bem definidas, principalmente as relacionadas à adequação do teste de germinação. Objetivou-se testar substratos e temperaturas para realização do teste de germinação em sementes de Acca sellowiana (O. Berg) Burret. (goiaba-serrana), Campomanesia xanthocarpa O. Berg (guabiroba), Eugenia involucrata DC. (cereja-do-mato) e Eugenia pyriformis Camb. (uvaia). Sementes de diferentes procedências foram submetidas ao teste de germinação em substratos areia e rolo de papel tipo germitest, umedecidos com água destilada, e nas temperaturas $15{ }^{\circ} \mathrm{C}, 25^{\circ} \mathrm{C}, 30^{\circ} \mathrm{C}$, $35^{\circ} \mathrm{C}$ e $20-30{ }^{\circ} \mathrm{C}$, sob luz constante, em germinadores tipo BOD. O delineamento experimental utilizado foi o inteiramente casualizado com quatro repetições de 25 sementes/tratamento/lote/espécie, e as médias dos tratamentos foram comparadas pelo teste de Tukey $(\mathrm{P}<0,01)$. O teste de germinação pode ser conduzido na temperatura de $25^{\circ} \mathrm{C}$ e em substrato rolo de papel para Eugenia involucrata e Eugenia pyriformis. Ambos os substratos podem ser utilizados para o teste de germinação de Acca sellowiana, a $25^{\circ} \mathrm{C}$. A temperatura de $25^{\circ} \mathrm{C}$ e alternância de $20-30^{\circ} \mathrm{C}$ são indicadas para Campomanesia xanthocarpa, assim como, os substratos areia e rolo de papel.

Palavras-chave: Acca sellowiana; Campomanesia xanthocarpa; Eugenia involucrata; Eugenia pyriformis.

\section{ABSTRACT}

The Brazilian species of the Myrtaceae comprises several arborescent and shrubby plants that are used to produce fruit for fresh consumption or industrialization. Information about the seed quality evaluations for these species are scarce in the literature, mainly related to the adequacy of the germination tests. This study tests different substrates and temperatures to test the germination of Acca sellowiana (O. Berg) Burret. (goiaba-serrana), Campomanesia xanthocarpa O. Berg (guabiroba), Eugenia involucrata DC. (cereja-domato) and Eugenia pyriformis Camb. (uvaia) seeds. Two seed lots were collected at different locations, one for each species, according to the requirements of the selection matrices. Sand and germitest paper towel rolls substrates were used, moistened with distilled water, and submitted to temperatures of $15{ }^{\circ} \mathrm{C}$, $25^{\circ} \mathrm{C}, 30^{\circ} \mathrm{C}, 35^{\circ} \mathrm{C}$ and $20-30^{\circ} \mathrm{C}$ under constant light, on P.D.A. germinators. The experimental delineation was entirely randomized with four repetitions of 25 seeds/treatment/lot/specie, and treatment means were

1 Engenheiro Florestal, Doutorando em Produção Vegetal, Centro de Ciências Agroveterinárias, Universidade do Estado de Santa Catarina, Av. Luiz de Camões, 2090, Bairro Conta Dinheiro, CEP 88520-000, Lages (SC), Brasil. julianopgomes@yahoo.com.br

2 Engenheira Florestal, Dr ${ }^{\mathrm{a}}$., Professora Adjunto do Departamento de Engenharia Florestal, Centro de Ciências Agroveterinárias, Universidade do Estado de Santa Catarina, Av. Luis de Camões, 2090, CEP 88520-000, Lages (SC),Brasil.A21mo@cav.udesc.br

3 Engenheira Agrônoma, Doutoranda em Produção Vegetal, Centro de Ciências Agroveterinárias, Universidade do Estado de Santa Catarina, Av. Luiz de Camões, 2090, Bairro Conta Dinheiro, CEP 88520-000, Lages (SC), Brasil. paulaiaschitzki@hotmail.com

4 Engenheiro Florestal, Mestre em Produção Vegetal, Centro de Ciências Agroveterinárias, Universidade do Estado de Santa Catarina, Av. Luiz de Camões, 2090, Bairro Conta Dinheiro, CEP 88520-000, Lages (SC), Brasil. felipe@florestal.eng.br

Recebido para publicação em 22/11/2011 e aceito em 13/05/2014

Ci. Fl., v. 26, n. 1, jan.-mar., 2016 
compared using the Tukey test $(\mathrm{P}<0.01)$. The germination tests can be conducted at $25{ }^{\circ} \mathrm{C}$ using paper roll substrate for Eugenia pyriformis and E. involucrate. Both substrates can be used to Acca sellowiana germination test at $25^{\circ} \mathrm{C}$. The temperature of $25^{\circ} \mathrm{C}$ and alternating $20-30^{\circ} \mathrm{C}$ are indicated for Campomanesia xanthocarpa, as well as sand and paper roll substrates.

Keywords: Acca sellowiana; Campomanesia xanthocarpa; Eugenia involucrata; Eugenia pyriformis.

\section{INTRODUÇÃO}

Frutíferas nativas pertencentes à família Myrtaceae são amplamente distribuídas pelo território brasileiro e constituem um patrimônio genético de grande valor. Além de apresentarem potencial para a exploração agrícola (LATTUADA et al., 2010), também são um importante recurso para restauração de áreas antropizadas. Esta família botânica é considerada uma das mais importantes da flora brasileira, com 23 gêneros (LANDRUM e KAWASAKI, 1997) e aproximadamente 820 espécies nativas e subespontâneas (BARROSO et al., 1984). A distribuição das espécies ocorre por todos os continentes, com exceção da Antártica, e com nítida predominância nas regiões tropicais e subtropicais (BARROSO, 1991; MARCHIORI e SOBRAL, 1997). É frequentemente citada como um dos grupos lenhosos dominantes em diversas formações naturais, sobretudo na Mata Atlântica (REITZ et al., 1978; GUILHERME et al., 2004).

Atualmente há uma crescente demanda dos mercados interno e externo por produtos à base de frutas nativas e de sabor exótico. Neste contexto, as espécies brasileiras da família Myrtaceae compreendem diversas plantas, arbóreas e arbustivas, que podem ser utilizadas na produção de frutos para consumo in natura ou para industrialização (LORENZI, 1992, 1998; DONADIO e MORO, 2004), como a Acca sellowiana (O. Berg) Burret. (goiaba-serrana), Campomanesia xanthocarpa $\mathrm{O}$. Berg (guabiroba), Eugenia involucrata DC. (cerejado-mato) e Eugenia pyriformis Camb. (uvaia).

Devido à importância das espécies dessa família, tanto para recomposição ambiental quanto para comercialização de produtos, a demanda por sementes ou mudas de espécies florestais nativas vem sendo crescente. Considerando-se que a grande maioria dessas espécies é propagada via sexuada, o sucesso na formação das mudas depende da qualidade das sementes utilizadas (REGO et al., 2009).

O principal teste utilizado para avaliação da qualidade de sementes, de forma geral, é o de germinação. Entretanto, as informações existentes na literatura sobre análise da qualidade de sementes de espécies florestais são inexpressivas frente às culturas agrícolas, como pode ser observado nas Regras para Análise de Sementes (BRASIL, 2009). $\mathrm{O}$ teste de germinação é realizado em condições favoráveis de luz, água, temperatura e substrato, as quais dependem da espécie.

$\mathrm{O}$ substrato influencia o processo germinativo em função de sua estrutura, capacidade de retenção de água, aeração, grau de infestação de patógenos, superfície de contato, dentre outros (NASCIMENTO et al., 2003). Pereira e Andrade (1994) recomendaram o uso de vermiculita, papel de filtro ou papel-toalha para o teste de germinação de sementes de Psidium guajava L.. Guimarães et al. (2007) testaram a germinação de Calyptranthes clusiifolia (Miq.) O. Berg. em quatro substratos (areia, substrato agrícola "Mecplant", vermiculita e rolo de papel) e constataram que o único substrato não eficiente foi o rolo de papel.

Já a temperatura interfere na germinação, a partir da sua influência sobre a velocidade de absorção de água e por afetar as reações bioquímicas (CARVALHO e NAKAGAWA, 1983). Para algumas espécies o desempenho germinativo das sementes é favorecido por temperaturas constantes, como em Eugenia rostrifolia D. Legrand (SANTOS et al., 2004), enquanto para outras o ideal é a alternância de temperatura, a exemplo de Caryophyllus aromaticum L. (MAEDA et al., 1991) e, ainda, observam-se insensibilidade ao regime de temperatura utilizado, como foi verificado nas sementes de Campomanesia adamantium Camb. (SCALON et al., 2009).

Objetivou-se, neste trabalho, testar a influência de substratos e temperaturas sobre o teste de germinação de sementes de Acca sellowiana (goiaba-serrana), Campomanesia xanthocarpa (guabiroba), Eugenia involucrata (cereja-do-mato) e Eugenia pyriformis (uvaia).

\section{MATERIAL E MÉTODOS}

Frutos maduros, de acordo com a coloração característica da espécie, de Acca sellowiana, 
Campomanesia xanthocarpa, Eugenia involucrata e Eugenia pyriformis foram coletados em diferentes matrizes, na região do Planalto Catarinense, nos anos de 2009 e 2010 (Tabela 1). Os frutos foram submetidos ao processo de beneficiamento (despolpamento, lavagem em água corrente e secagem), utilizando-se os procedimentos indicados para cada espécie, descritos por Lorenzi (1992) e Silva et al. (1993).

A caracterização inicial dos lotes foi realizada por meio da determinação do teor de água, utilizando-se o método de estufa a $105 \pm 3{ }^{\circ} \mathrm{C}$ por 24 horas, segundo metodologia descrita nas Regras para Análise de Sementes (BRASIL, 2009).

As sementes foram submetidas ao teste de germinação, em esquema fatorial 5 × 2 (Temperatura $\mathrm{x}$ Substrato), sendo testados os tratamentos sobre areia (SA) e rolo de papel (RP) tipo germitest, umedecidos com água destilada com volume $(\mathrm{mL})$ equivalente a 3,0 vezes o peso do substrato $(\mathrm{g})$, e temperaturas de $15{ }^{\circ} \mathrm{C}, 25{ }^{\circ} \mathrm{C}, 20-30{ }^{\circ} \mathrm{C}, 30^{\circ} \mathrm{C}$ e $35^{\circ} \mathrm{C}$ sob luz constante, em germinadores tipo BOD.

A avaliação da germinação foi realizada diariamente a partir do início da protrusão radicular. Após um mês de germinação, as avaliações foram realizadas semanalmente, até a estabilização de todos os estandes, que, em alguns tratamentos, se estenderam até os 143 dias para Acca sellowiana, 58 dias para Campomanesia xanthocarpa, 49 dias para Eugenia involucrata e 126 dias para Eugenia pyriformis. A classificação das plântulas como normais ou anormais foi realizada de acordo com as Regras para Análise de Sementes (BRASIL, 2009), considerando normais as plântulas com todas as estruturas essenciais em perfeito desenvolvimento. Também foi determinada a análise de vigor por meio da primeira contagem de germinação, realizada após 15 dias de semeadura.

O delineamento experimental utilizado foi o inteiramente casualizado, com quatro repetições de 25 sementes por tratamento para cada lote, e as médias dos tratamentos foram comparadas pelo teste de Tukey $(\mathrm{P}<0,01)$.

\section{RESULTADOS E DISCUSSÃO}

Os lotes de sementes de Eugenia involucrata apresentaram umidade média de 50\%, valor que pode ser considerado relativamente baixo, quando comparado com estudos realizados por Feltrin et al. (1991), Barbedo et al. (1998) e Maluf et al. (2003), os quais encontraram teores de água entre 60 e $80 \%$. Delgado e Barbedo (2007) também verificaram elevados conteúdos de água ( $>40 \%)$ em sementes de espécies brasileiras do gênero Eugenia após o beneficiamento, destacando-se como uma característica inerente às espécies pertencentes a este grupo.

As sementes de Campomanesia xanthocarpa, de ambos os lotes, apresentaram valor médio de $54 \%$ de umidade. Pode-se dizer que ocorre expressiva variação quanto ao grau de umidade em sementes de espécie do mesmo gênero, pois, segundo Santos et al. (2004), o teor de água nas sementes de Campomanesia guazumifolia (Cambes.) O. Berg. foi de $9 \%$.

O valor médio de umidade das sementes de Eugenia pyriformis após o beneficiamento foi de $51 \%$, sendo superior ao encontrado por Scalon et al. (2012), que foi de $45 \%$, no qual foram classificadas como recalcitrantes. Com relação à Acca sellowiana, o valor de umidade de $33 \%$ foi considerado o menor quando comparado às demais espécies estudadas, porém, similar ao resultado encontrado por Gomes et al. (2013), o qual foi de $36 \%$. O menor valor de umidade das sementes de Acca sellowiana, talvez, esteja relacionado ao comportamento fisiológico das mesmas, pois, segundo Gomes et al. (2013),

TABELA 1: Espécies e procedências dos lotes de sementes analisados.

TABLE 1: Species and origins of seeds lots analyzed.

\begin{tabular}{clccc}
\hline Lotes & \multicolumn{1}{c}{ Nome Científico } & Nome Comum & Local de Coleta & Data de Coleta \\
\hline 1 & Acca sellowiana (Berg.) Burret & Goiaba-serrana & Correia Pinto/SC & abr/10 \\
2 & Acca sellowiana (Berg.) Burret & Goiaba-serrana & Painel/SC & abr/10 \\
1 & Campomanesia xanthocarpa Berg. & Guabiroba & Correia Pinto/SC & dez/09 \\
2 & Campomanesia xanthocarpa Berg. & Guabiroba & Ponte Alta/SC & dez/09 \\
1 & Eugenia involucrata DC & Cereja-do-mato & Correia Pinto/SC & nov/09 \\
2 & Eugenia involucrata DC & Cereja-do-mato & Lages/SC & nov/09 \\
1 & Eugenia pyriformis Camb. & Uvaia & Correia Pinto/SC & jan $/ 10$ \\
2 & Eugenia pyriformis Camb. & Uvaia & Ponte Alta/SC & jan $/ 10$ \\
\hline
\end{tabular}


esta espécie apresenta sementes fisiologicamente classificadas como intermediárias.

Em relação ao teste de germinação, para todas as espécies estudadas, não foi observada interação significativa entre os fatores temperatura e substrato para o parâmetro primeira contagem de germinação; observou-se que as maiores porcentagens de germinação, na primeira contagem, foram obtidas sob a temperatura de $25{ }^{\circ} \mathrm{C}$; em relação aos substratos, foi verificado que o rolo de papel foi propício para expressar o vigor das sementes. (Tabela 2).

No teste de germinação de sementes de Eugenia involucrata observou-se que os resultados médios obtidos nos diferentes tratamentos diferiram entre si pelo teste de médias. Houve maior porcentagem de germinação quando se utilizou a temperatura de $25{ }^{\circ} \mathrm{C}$, para ambos os lotes (Tabela 3). Para germinação de sementes de espécies da Mata Atlântica, a temperatura ótima mais frequente é de $25^{\circ} \mathrm{C}$. Esse valor parece estar relacionado à temperatura característica deste bioma, já que nesta região há predominância de temperaturas mais amenas. Tal constatação reforça a hipótese de que a temperatura ótima de germinação está relacionada às temperaturas da região de origem da espécie na época favorável para a germinação (ANDRADE e FERREIRA, 2000), constituindo-se em uma adaptação fisiológica. A baixa germinação na temperatura de $35{ }^{\circ} \mathrm{C}$ pode ter relação com o efeito de altas temperaturas na germinação, já que, nestas condições, alterações enzimáticas podem ocorrer, aumentando a exigência por oxigênio e por consequência, acelerando a velocidade respiratória das sementes (CARVALHO e NAKAGAWA, 2000).

A germinação em rolos de papel tipo germitest apresentou desempenho superior quando comparado com o substrato sobre areia (Tabela 3). A areia apresenta fácil drenagem da água (DOUSSEAU et al., 2011), o que provoca o ressecamento da parte superior do substrato e, com isto, pode prejudicar a germinação das sementes de muitas espécies, como verificado em sementes de Campomanesia phaea (Berg.) Landr. (MALUF e PISCIOTTANOEREIO, 2005), entretanto, para Campomanesia pubescens, o menor conteúdo de água no substrato areia favoreceu a germinação. Além disso, por se tratar de um material mais denso, torna trabalhoso o manuseio no germinador (FIGLIOLIA et al., 1993). Desta forma, a utilização de substrato papel, na forma de rolo, é a opção mais apropriada para o teste de germinação de Eugenia involucrata (Tabela 3) e Campomanesia xanthocarpa (Tabela 4).

Os resultados do teste de germinação das sementes de Campomanesia xanthocarpa apresentaram interação significativa entre temperatura e substrato (Tabela 4).

As temperaturas de $25^{\circ} \mathrm{C}$ e $20-30^{\circ} \mathrm{C}$ proporcionaram percentuais mais altos de germinação para ambos os lotes, independente do substrato utilizado, corroborando com os estudos de Dousseau et al. (2011), que verificaram que sementes de Campomanesia pubescens (DC.) O. Berg.

TABELA 2: Primeira contagem de germinação (\%) em sementes de Eugenia involucrata, Campomanesia xanthocarpa, Acca sellowiana e Eugenia pyriformis, em diferentes temperaturas e substratos.

TABLE 2: First germination counting (\%) of Eugenia involucrata, Campomanesia xanthocarpa, Acca sellowiana e Eugenia pyriformis seeds, at different temperatures and substrates.

\begin{tabular}{ccccccccc}
\hline \multirow{2}{*}{ Temperaturas } & \multicolumn{2}{c}{ Eugenia involucrata } & \multicolumn{2}{c}{ Campomanesia xanthocarpa } & \multicolumn{2}{c}{ Acca sellowiana } & \multicolumn{2}{c}{ Eugenia pyriformis } \\
\cline { 2 - 8 } & Lote 1 & Lote 2 & Lote 1 & Lote 2 & Lote 1 & Lote 2 & Lote 1 & Lote 2 \\
\hline $15{ }^{\circ} \mathrm{C}$ & $4 \mathrm{c}$ & $0 \mathrm{c}$ & $4 \mathrm{~d}$ & $0 \mathrm{c}$ & $0 \mathrm{c}$ & $0 \mathrm{~d}$ & $4 \mathrm{c}$ & $8 \mathrm{c}$ \\
$25{ }^{\circ} \mathrm{C}$ & $8 \mathrm{bc}$ & $12 \mathrm{~b}$ & $12 \mathrm{~b}$ & $12 \mathrm{ab}$ & $12 \mathrm{~b}$ & $16 \mathrm{bc}$ & $8 \mathrm{~b}$ & $16 \mathrm{~b}$ \\
$20 / 30^{\circ} \mathrm{C}$ & $16 \mathrm{a}$ & $24 \mathrm{a}$ & $28 \mathrm{a}$ & $16 \mathrm{a}$ & $20 \mathrm{a}$ & $24 \mathrm{a}$ & $16 \mathrm{a}$ & $24 \mathrm{a}$ \\
$30^{\circ} \mathrm{C}$ & $12 \mathrm{~b}$ & $12 \mathrm{~b}$ & $\mathrm{a} 6 \mathrm{~b}$ & $12 \mathrm{ab}$ & $12 \mathrm{~b}$ & $16 \mathrm{bc}$ & $12 \mathrm{ab}$ & $16 \mathrm{~b}$ \\
$35^{\circ} \mathrm{C}$ & $4 \mathrm{c}$ & $8 \mathrm{~b}$ & $8 \mathrm{c}$ & $8 \mathrm{~b}$ & $4 \mathrm{~b}$ & $12 \mathrm{c}$ & $8 \mathrm{~b}$ & $8 \mathrm{c}$ \\
\hline Substratos & Lote 1 & Lote 2 & Lote 1 & Lote 2 & Lote 1 & Lote 2 & Lote 1 & Lote 2 \\
\hline RP & $16 \mathrm{a}$ & $20 \mathrm{a}$ & $24 \mathrm{a}$ & $16 \mathrm{a}$ & $16 \mathrm{a}$ & $20 \mathrm{a}$ & $12 \mathrm{a}$ & $20 \mathrm{a}$ \\
SA & $8 \mathrm{~b}$ & $12 \mathrm{~b}$ & $16 \mathrm{~b}$ & $8 \mathrm{~b}$ & $8 \mathrm{~b}$ & $16 \mathrm{~b}$ & $8 \mathrm{~b}$ & $12 \mathrm{~b}$ \\
\hline
\end{tabular}

Em que: Médias seguidas de mesma letra não diferem significativamente pelo teste de Tukey ao nível de 1\% de significância. $\mathrm{CV}=17,96 \%, \mathrm{~F}=6,43(\mathrm{P}=0,0058) . \mathrm{RP}=$ rolo de papel: $\mathrm{SA}=$ sobre areia. 
TABELA 3: Germinação (\%) de sementes de Eugenia involucrata, em diferentes temperaturas e substratos.

TABLE 3: Germination (\%) of Eugenia involucrata seeds, at different temperatures and substrates.

\begin{tabular}{|c|c|c|}
\hline \multirow{2}{*}{ Temperaturas $\left({ }^{\circ} \mathrm{C}\right)$} & \multicolumn{2}{|c|}{ Lotes } \\
\hline & 1 & 2 \\
\hline 15 & $0 \mathrm{C}$ & $2 \mathrm{D}$ \\
\hline 25 & $66 \mathrm{~A}$ & $93 \mathrm{~A}$ \\
\hline $20 / 30$ & $56 \mathrm{~A}$ & $72 \mathrm{~B}$ \\
\hline 30 & $33 \mathrm{~B}$ & $63 \mathrm{~B}$ \\
\hline 35 & $34 \mathrm{~B}$ & $48 \mathrm{C}$ \\
\hline Substrato & 1 & 2 \\
\hline $\mathrm{RP}$ & $38 \mathrm{~A}$ & $64 \mathrm{~A}$ \\
\hline SA & $36 \mathrm{~A}$ & $47 \mathrm{~B}$ \\
\hline CV $(\%)$ & 34,3 & 12,6 \\
\hline $\mathrm{F}$ & 14.68 & 95.31 \\
\hline
\end{tabular}

Em que: Médias seguidas pela mesma letra não diferem entre si pelo teste de Tukey a $1 \%$ de probabilidade. $\mathrm{RP}=$ rolo de papel: $\mathrm{SA}=$ sobre areia.

apresentaram maior porcentagem de germinação sob temperatura de $25{ }^{\circ} \mathrm{C}$ e $20-30{ }^{\circ} \mathrm{C}$. Além dessas temperaturas, quando o substrato utilizado foi rolo de papel, as temperaturas que possibilitaram melhores resultados de germinação foram $30{ }^{\circ} \mathrm{C}$ e $35^{\circ} \mathrm{C}$. De acordo com Dias et al. (2011), sementes de Myrciaria cauliflora Berg. apresentam melhor desempenho germinativo sob temperatura de $30^{\circ} \mathrm{C}$. Foi verificado que sementes de Campomanesia xanthocarpa apresentam melhores resultados de germinação quando utilizadas temperaturas variando de 15 a $30^{\circ} \mathrm{C}$ (SANTOS et al., 2004). Vale ressaltar, que os autores citados utilizaram a protrusão radicular como parâmetro de avaliação do teste de germinação, esta condição, provavelmente auxiliou o aumento expressivo da porcentagem de germinação sob ampla variação térmica.

Interação significativa entre temperatura e substrato para sementes de Amburana cearensis (Allemão) A. C. Smith foi relatada por Guedes et al. (2010), explicando que a capacidade de retenção de água e a quantidade de luz que o substrato permite chegar à semente podem ser responsáveis por diferentes respostas obtidas até para a mesma temperatura.

Para Acca sellowiana, foi possível verificar que o potencial germinativo se alterou conforme os tratamentos utilizados, e que, para o lote 2 e em temperatura de $30{ }^{\circ} \mathrm{C}$ ou superior, houve interação significativa entre os fatores temperatura e substrato (Tabela 5).

Para o substrato rolo de papel, as temperaturas ideais foram $25{ }^{\circ} \mathrm{C}, 20-30{ }^{\circ} \mathrm{C}$ e $30{ }^{\circ} \mathrm{C}$, no entanto, quando o teste de germinação foi realizado em areia, apenas a temperatura de $25^{\circ} \mathrm{C}$ foi favorável. As diferenças encontradas na germinação de lotes de procedências distintas da mesma espécie podem ocorrer devido à variabilidade genética das

TABELA 4: Germinação (\%) de sementes de Campomanesia xanthocarpa, em diferentes temperaturas e substratos.

TABLE 4: Germination (\%) of Campomanesia xanthocarpa seeds, at different temperatures and substrates.

\begin{tabular}{|c|c|c|c|c|}
\hline \multirow{3}{*}{ Temperaturas $\left({ }^{\circ} \mathrm{C}\right)$} & \multicolumn{4}{|c|}{ Lotes } \\
\hline & \multicolumn{2}{|c|}{1} & \multicolumn{2}{|c|}{2} \\
\hline & \multicolumn{4}{|c|}{ Substratos } \\
\hline & $\mathrm{RP}$ & SA & $\mathrm{RP}$ & SA \\
\hline 15 & $23 \mathrm{Ab}$ & $16 \mathrm{Ab}$ & $7 \mathrm{Ab}$ & $7 \mathrm{Abc}$ \\
\hline 25 & $72 \mathrm{Aa}$ & $66 \mathrm{Aa}$ & $74 \mathrm{Aa}$ & $63 \mathrm{Aa}$ \\
\hline $20 / 30$ & $76 \mathrm{Aa}$ & $78 \mathrm{Aa}$ & $73 \mathrm{Aa}$ & $44 \mathrm{Aab}$ \\
\hline 30 & $74 \mathrm{Aa}$ & $61 \mathrm{Aa}$ & $69 \mathrm{Aa}$ & $28 \mathrm{Bbc}$ \\
\hline 35 & $87 \mathrm{Aa}$ & $23 \mathrm{Bb}$ & $74 \mathrm{Aa}$ & $20 \mathrm{Bbc}$ \\
\hline CV $(\%)$ & \multicolumn{2}{|c|}{22,8} & \multicolumn{2}{|c|}{27,3} \\
\hline $\mathrm{F}$ & \multicolumn{2}{|c|}{16,3} & \multicolumn{2}{|c|}{20,3} \\
\hline
\end{tabular}

Em que: Médias seguidas pela mesma letra minúscula nas colunas e maiúscula nas linhas não diferem entre si pelo teste de Tukey a $1 \%$ de probabilidade. $\mathrm{RP}=$ rolo de papel: $\mathrm{SA}=$ sobre areia. 
espécies florestais silvestres (WIELEWICKI et al., 2006), ou ainda, pelas variações climáticas as quais as matrizes são submetidas durante sua formação.

Para Acca sellowiana, a qual ocorre naturalmente em elevadas altitudes, esperavase que a temperatura de $15{ }^{\circ} \mathrm{C}$ fosse um fator favorável ao processo germinativo, no entanto, foi possível verificar que, assim como as demais espécies estudadas, a temperatura ideal para testar a viabilidade das sementes foi $25^{\circ} \mathrm{C}$, independente do substrato (Tabela 5). Estes resultados corroboram aos estudos realizados para várias espécies nativas como de Dresch et al. (2012), que verificaram que a melhor temperatura para realizar o teste de germinação em Campomanesia adamantium (Camb.) O. Berg foi de $25^{\circ} \mathrm{C}$. Em relação ao substrato, para o lote 1 não foram observadas diferenças significativas entre a areia e o rolo de papel (Tabela 6).

Nas sementes de Eugenia pyriformis, o desempenho germinativo de ambos os lotes foi melhor quando as sementes foram submetidas à temperatura de $25{ }^{\circ} \mathrm{C}$, em contrapartida, sob temperatura de $15{ }^{\circ} \mathrm{C}$ a germinação sofreu perdas acentuadas na porcentagem total de plântulas normais (Tabela 7). Carvalho e Nakagawa (2000) afirmaram que temperaturas abaixo do ótimo tendem a reduzir o porcentual de germinação. $\mathrm{O}$ substrato mais indicado para o teste de germinação de sementes de Eugenia pyriformis foi rolo de

TABELA 5: Germinação (\%) de sementes de Acca sellowiana, em diferentes temperaturas e substratos.

TABLE5: Germination(\%) of Acca sellowiana seeds, at different temperatures and substrates.

\begin{tabular}{ccc}
\hline \multirow{2}{*}{ Temperaturas $\left({ }^{\circ} \mathrm{C}\right)$} & \multicolumn{2}{c}{ Lote 2} \\
\cline { 2 - 3 } & \multicolumn{2}{c}{ Substratos } \\
\hline & $\mathrm{RP}$ & $\mathrm{SA}$ \\
25 & $2 \mathrm{Ac}$ & $2 \mathrm{Ad}$ \\
$20 / 30$ & $96 \mathrm{Aa}$ & $90 \mathrm{Aa}$ \\
30 & $76 \mathrm{Aab}$ & $69 \mathrm{Ab}$ \\
35 & $81 \mathrm{Aab}$ & $45 \mathrm{Bc}$ \\
$\mathrm{CV}(\%)$ & $65 \mathrm{Ab}$ & $31 \mathrm{Bc}$ \\
$\mathrm{F}$ & \multicolumn{3}{c}{12,6} \\
\hline \multicolumn{2}{c}{95,3} \\
\hline
\end{tabular}

Em que: Médias seguidas pela mesma letra minúscula nas colunas e maiúscula nas linhas não diferem entre si pelo teste de Tukey a $1 \%$ de probabilidade. $\mathrm{RP}=$ rolo de papel: $\mathrm{SA}=$ sobre areia. papel, para ambos os lotes, no entanto, o substrato areia também demonstrou desempenho satisfatório para o lote 2 .

Provavelmente a melhor germinação em rolo de papel seja consequência da maior capacidade de retenção de umidade e área de contato com as sementes. Segundo Amaral (1986), nos testes de germinação o substrato deve permanecer suficientemente umedecido durante o período de duração do teste, mas nunca envolvendo as sementes com uma película de água, que pode restringir a respiração das mesmas.

Silva et al. (2005) testaram a temperatura de $30^{\circ} \mathrm{C}$ para germinação de sementes de Eugenia uniflora L., Eugenia involucrata DC. e Eugenia brasiliensis Lam., que, segundo Valio e Ferreira (1992); Landgraf (1994) e Dignart (1998), está dentro da faixa considerada ótima para diversas espécies florestais nativas. No entanto, para as espécies estudadas, independentemente do substrato utilizado, a temperatura de $25^{\circ} \mathrm{C}$ foi a que possibilitou melhor desempenho de germinação, condizendo com estudos que declaram que a faixa entre 20 e $30^{\circ} \mathrm{C}$ é a mais adequada para germinação das sementes da maioria das espécies tropicais e subtropicais (BORGES e RENA, 1993; SILVA e AGUIAR, 1998).

TABELA 6: Germinação (\%) de sementes de Acca sellowiana, em diferentes temperaturas e substratos.

TABLE 6: Germination (\%) of Acca sellowiana seeds, at different temperatures and substrates.

\begin{tabular}{cc}
\hline Temperaturas $\left({ }^{\circ} \mathrm{C}\right)$ & Lote 1 \\
\hline 15 & $0 \mathrm{C}$ \\
25 & $90 \mathrm{~A}$ \\
$20 / 30$ & $72 \mathrm{~B}$ \\
30 & $64 \mathrm{~B}$ \\
35 & $57 \mathrm{~B}$ \\
\hline Substratos & Lote 1 \\
\hline RP & $53 \mathrm{~A}$ \\
SA & $60 \mathrm{~A}$ \\
CV $(\%)$ & 17,7 \\
F & 41 \\
\hline
\end{tabular}

Em que: Médias seguidas pela mesma letra minúscula nas colunas e maiúscula nas linhas não diferem entre si pelo teste de Tukey a $1 \%$ de probabilidade. $\mathrm{RP}=$ rolo de papel: $\mathrm{SA}=$ sobre areia. 
TABELA 7: Germinação (\%) de sementes de Eugenia pyriformis, em diferentes substratos.

TABLE 7: Germination (\%) of Eugenia pyriformis seeds, at different temperatures and substrates

\begin{tabular}{ccc}
\hline \multirow{2}{*}{ Temperatura $\left({ }^{\circ} \mathrm{C}\right)$} & \multicolumn{2}{c}{ Lotes } \\
\cline { 2 - 3 } & 1 & 2 \\
\hline 15 & $0 \mathrm{C}$ & $2 \mathrm{D}$ \\
25 & $92 \mathrm{~A}$ & $93 \mathrm{~A}$ \\
$20 / 30$ & $56 \mathrm{~A}$ & $72 \mathrm{~B}$ \\
30 & $33 \mathrm{~B}$ & $63 \mathrm{~B}$ \\
35 & $34 \mathrm{~B}$ & $48 \mathrm{C}$ \\
\hline Substratos & 1 & 2 \\
\hline RP & $52 \mathrm{~A}$ & $58 \mathrm{~A}$ \\
SA & $44 \mathrm{~B}$ & $55 \mathrm{~A}$ \\
CV $(\%)$ & 13,7 & 18 \\
F & 95,6 & 58 \\
\hline
\end{tabular}

Em que: Médias seguidas pela mesma letra não diferem entre si pelo teste de Tukey a $1 \%$ de probabilidade. $\mathrm{RP}=$ rolo de papel: $\mathrm{SA}=$ sobre areia.

\section{CONCLUSÃO}

O teste de germinação pode ser conduzido na temperatura de $25^{\circ} \mathrm{C}$ e em substrato rolo de papel para Eugenia involucrata e Eugenia pyriformis; a $25{ }^{\circ} \mathrm{C}$ em substratos areia e rolo de papel para Acca sellowiana; e com alternância de temperatura de 20-30 ${ }^{\circ} \mathrm{C}$ ou $25{ }^{\circ} \mathrm{C}$ em substratos rolo de papel e areia para Campomanesia xanthocarpa.

\section{REFERÊNCIAS BIBLIOGRÁFICAS}

AMARAL, D. M. I. Padronização de testes em laboratório com sementes florestais. In: SIMPÓSIO BRASILEIRO SOBRE TECNOLOGIA DE SEMENTES FLORESTAIS, 1., 1984, Belo Horizonte. Anais... Brasília: ABRATES, 1986. p. 267-283.

ANDRADE, R. N. B. de; FERREIRA, A. G. Germinação e armazenamento de sementes de uvaia (Eugenia pyriformis Camb.) - Myrtaceae. Revista Brasileira de Sementes, Brasília, v. 22, n. 2, p. 118-125, 2000.

ANDRADE, A.C.S.; EREIRA, T.S. Efeito do Substrato e da Temperatura na Germinação e no Vigor de Sementes de Cedro - Cedrela odorata L. (MELIACEAE). Revista Brasileira de Sementes, Brasília, vol. 16, no 1, p. 34-40, 1994.
BARBEDO, C.J.et al. Germinação e armazenamento de diásporos de cerejeira (Eugenia involucrata DC - Myrtaceae) em função do teor de água. Revista Brasileira de Sementes, Brasília, v.20, n.1, p. 184-188, 1998.

BARROSO, G.M. Sistemática de angiospermas do Brasil. Viçosa: UFV, 1991. v.3, 326p.

BARROSO, G. $M$. et al. Sistemática de angiospermas do Brasil, Viçosa: UFV, 1984. v. 2,377 p.

BEZERRA, J. E. F. et al. Pitanga (Eugenia uniflora L.), Jaboticabal: FUNEP, 2000. 30p. (Série Frutas Nativas, 1).

BORGES, E.E.L.; RENA,A.B. 1993. Germinação de sementes. In: AGUIAR, I.B.; PIÑA-RODRIGUES, F.C.M.; FIGLIOLIA, M.B. (coord.) Sementes florestais tropicais. Brasília, ABRATES. P.83-136. BRASIL. Ministério da Agricultura e Reforma Agrária. Regras para análise de sementes. Brasília: Mapa/ACS, 2009.399 p.

CARVALHO, N. M.; NAKAGAWA, J. Sementes: ciência, tecnologia e produção. 4.ed. Jaboticabal: FUNEP, 2000. 588p.

CARVALHO, N.M., NAKAGAWA, J. Sementes: ciência, tecnologia e produção. Campinas: Fundação Cargil, 1983. 429p.

DELGADO, L. F.; BARBEDO, C. J. Tolerância à dessecação de sementes de espécies de Eugenia. Pesquisa agropecuária brasileira, Brasília, v.42, n.2, p. 265-272, fev. 2007.

DIAS et al. Influência da temperatura e substrato na germinação de sementes de jabuticabeira (Myrciaria cauliflora Berg.), IDESIA, Chile, v. 29, n 1, 2011

DIGNART, S. Análise de sementes de jatobá do cerrado [Hymenaea stigonocarpa (Hayne) Mart.] e barbatimão [Stryphnodendron adstringens (Mart.) Cov.]. 1998, 58f. Dissertação (Mestrado em Agricultura Tropical) - Faculdade de Agronomia e Medicina Veterinária, Universidade Federal de Mato Grosso, Cuiabá-MT, 1998.

DONADIO, L. C.; MORO, F. V. Potential of Brazilian Eugenia (Myrtaceae) - as ornamental and as a fruit crop. Acta Horticulturae, Leuven, v.632, p.65-68, 2004.

DOUSSEAU, S. et al. Ecofisiologia da germinação de sementes de Campomanesia pubescens. Ciência Rural, Santa Maria. v.41, n.8, p.1362-1368, 2011. DRESCH et al. Germinação de sementes de Campomanesia adamantium (Camb.) O. Berg em diferentes temperaturas e umidades do substrato, Scientia Forestalis, Piracicaba, v. 40, n. 94, p. 223-229, 2012. 
FELTRIN, I. J. et al. Estudo da germinação de Eugenia involucrata DC. In: JORNADA DE PESQUISA DA UFSM, 1., 1991, Santa Maria. Anais... Santa Maria, Universidade Federal de Santa Maria, 1991. p. 312.

FIGLIOLIA, M.B. et al. Análise de Sementes. In: AGUIAR, I.B.de; PIÑA-RODRIGUES, F.C.M. e FIGLIOLIA, M.B. (coord.). Sementes florestais tropicais. Brasília: ABRATES, 1993. cap. 4, p.137-174.

GOMES, et al. Secagem e classificação de Sementes de Acca sellowiana (O. Berg) Burret Myrtaceae quanto à tolerância à dessecação e ao armazenamento. Floresta e Ambiente, v.20, n.2, p. 207-215, 2013.

GUEDES, R. S. et al. Substratos e temperaturas para testes de germinação e vigor de sementes de Amburana cearensis (Allemão) A.C. Smith. Revista Árvore, Viçosa, v.34, n.1, p.57-64, 2010.

GUILHERME, F.A.G. et al. Effects of flooding regime and woody bamboos on tree community dynamics in a section of tropical semideciduous forest in South-Eastern Brazil. Plant Ecology, Oxford, n.174, p.19-36,2004.

GUIMARÃES, D. M. et al. Influência de Diferentes Substratos e Níveis de Temperatura Sobre o Processo Germinativo de Sementes de Calyptranthes clusiifolia (Myrtaceae). Revista Brasileira de Biociências, Porto Alegre, v. 5, supl.2, p.816-818, 2007.

LANDRUM, L.R. ; KAWASAKI, M.L. The genera of Myrtaceae in Brazil $\tilde{n}$ an illustrated synoptic treatment and identification keys. Brittonia v.49, p.508-536,1997.

LATTUADA, D.S. et al. Enxertia herbácea em Myrtaceae nativas do Rio Grande do Sul. Revista Brasileira de Fruticultura. Jaboticabal, v. 32, n. 4, p. 1285-1288. 2010.

LANDGRAF, P. R. C. Germinação de sementes de guarea (Guarea guidonea (L.) Sleumer), maçaranduba (Persea pyrifolia) e peito de pombo (Tapirira guianeensis Aul.). 1994. 91f. Dissertação (Mestrado em Agronomia) - Escola Superior de Agricultura de Lavras, Lavras, 1994.

LORENZI, H. Árvores brasileiras: manual de identificação e cultivo de plantas arbóreas nativas do Brasil. Nova Odessa: Plantarum, 1992. 368p.

LORENZI, H. Árvores brasileiras: manual de identificação e cultivo de plantas arbóreas nativas do Brasil. 2.ed. São Paulo: Editora Plantarum,1998, v.1.

MAEDA, J. A. et al. Germinação de sementes de Craveiro-da-Índia: Efeito de temperatura, polpa do fruto e tratamento fungicida. Pesquisa Agropecuária Brasileira, v.26, n. 6, p. 893-899. 1991.

MALUF, A.M. et al. Drying and storage of Eugenia involucrata DC. Scientia Agricola, Piracicaba, v.60, p.471-475, 2003.

MALUF, A.M.; PISCIOTTANO-EREIO, W.A. Secagem e armazenamento de sementes de cambuci. Pesquisa Agropecuária Brasileira, Brasília, v.40, n.7, p.707-714, 2005.

MARCHIORI, J. N. C.; SOBRAL, M. Dendrologia das angiospermas: Myrtales. 6 ed. Santa Maria: Editora da UFSM, 1997. 304 p.

NASCIMENTO, W. M. O. et al. Temperatura e substrato para a germinação de sementes de Parkia platycephala Benth. (Leguminoseae-Mimosoideae). Revista Agropecuária Tropical, Cuiabá, v. 7, n. 1, p. 119-129, 2003.

PACHECO, M.V. et al. Germination and vigor of Dimorphandra mollis Benth. Seeds under different temperatures and substrates. Revista Árvore, Viçosa, v.34, n.2, p.205-213, 2010.

PEREIRA, T.S.; ANDRADE, C.S. Germinação de Psidium guajava L. e Passiflora edulis SimsEfeito da temperatura, substrato e morfologia do desenvolvimento pós-seminal. Revista Brasileira de Sementes, Brasília, v.16, n.1, p.58- 62, 1994.

RAMOS, M. B. P.; VARELA, V. P. Efeito da temperatura e do substrato sobre a germinação de sementes de visgueiro do igapó (Parkia discolor Benth) Leguminosae, Mimosoideae. Revista de Ciências Agrárias, Manaus, n. 39, p. 123-133, 2003.

REGO, S.S. et al. Germinação de sementes de Blepharocalyx salicifolius (H.B.K.) Berg. em diferentes substratos e condições de temperatura, luz e umidade. Revista Brasileira de Sementes, Brasília, v. 31, n.2, p.212-220, 2009.

REITZ, R. et al. Projeto madeira de Santa Catarina. Sellowwia, n.34/35, p. 525, 1978.

SANTOS, C.M.R. dos. et al. Características de frutos e germinação de sementes de seis espécies de Myrtaceae nativas do Rio Grande do Sul. Ciência Florestal, Santa Maria, v.14, n.2, p.13-20, 2004.

SCALON, Silvana de Paula Quintão; NEVES, Eliane Marques da Silva; MASETO, Tathiana Elisa and PEREIRA, Zefa Valdivina. Sensibilidade à dessecação e aoarmazenamento em sementes de Eugenia pyriformis Cambess. (uvaia). Revista Brasileira de Fruticultura, v.34, n.1, p. 269-276, 2012. 
SCALON, S.P.Q. et al. Germinação de sementes e crescimento inicial de mudas de Campomanesia adamantium Camb.: efeito da lavagem, temperatura e bioestimulantes. Revista Brasileira de Sementes, Pelotas, v.31, n.2, p.96-103, 2009.

SILVA, A; AGUIAR, I.B. Germinação de sementes de canela-preta (Ocotea catharinensis Mez - Lauraceae) sob diferentes condições de luz e temperatura. Revista Instituto Florestal, São Paulo, v.10, n.1, p. 17-22, 1998.

SILVA, E. A. A. da. et al. Exogenous gibberellins inhibit coffee (Coffea arabica cv. Rubi) seed germination and cause cell death in the embryo. Journal of Experimental Botany, Oxford, v. 56, n. 413, p. 1029-1038, 2005.
SILVA, A. et al. Secagem, extração e beneficiamento de semente. In: AGUIAR, I.B., PIÑA-RODRIGUES, F.C.M., FIGLIOLIA, M.B. (coord). Sementes florestais tropicais. Brasília: ABRATES, 1993. cap. 8, p. 303-332.

VALIO, I.F.M.; FERREIRA, Z.L. Germination of seeds of Myrciaria cauliflora (Mart.) Berg. (Myrtaceae). Revista Brasileira de Fisiologia Vegetal, Lavras, v.4, n.2, p.95-98, 1992.

WIELEWICKI, A. P. et al. Proposta de padrões de germinação e teor de água para sementes de algumas espécies florestais presentes na região sul do Brasil. Revista brasileira de sementes, Pelotas, v.28, n.3, p. 191-197, 2006. 